MATHEMATICS OF COMPUTATION

Volume 81, Number 278, April 2012, Pages 1163-1177

S $0025-5718(2011) 02515-9$

Article electronically published on July 21, 2011

\title{
EFFICIENT COMPUTATION OF CASTELNUOVO-MUMFORD REGULARITY
}

\author{
AMIR HASHEMI
}

\begin{abstract}
In this paper, we introduce the notion of a homogeneous ideal in quasi stable position (QSP); a new definition for the notion of generic coordinates to compute efficiently the Castelnuovo-Mumford regularity of a homogeneous ideal. This definition is simple to check, because it is tested on the initial ideal for the degree reverse lexicographic ordering. It is explicit, because we provide an algorithm to decide whether a monomial ideal is in QSP or not. The main result of this paper is that the Castelnuovo-Mumford regularity of an ideal in QSP is the maximal degree of the elements of its reduced Gröbner basis with respect to the reverse lexicographic ordering. We have implemented an algorithm in (the distributed library noether.lib of) Singular based on the above results for computing the Castelnuovo-Mumford regularity of a general ideal, and we evaluate its performance via some examples.
\end{abstract}

\section{INTRODUCTION}

Let us consider a homogeneous ideal $I$ over the ring $R=K\left[x_{0}, \ldots, x_{n}\right]$ where $K$ is an arbitrary field. A good measure to estimate the complexity of the computation of the Gröbner basis of $I$ is the maximal degree of the polynomials which appear during the computation (see [11, 14, 15]). On the other hand, let

$$
0 \longrightarrow F_{\ell} \longrightarrow \cdots \longrightarrow F_{0} \longrightarrow I \longrightarrow 0
$$

be a minimal graded free resolution of $I$ and $e_{i}$ the maximal degree of the generators of $F_{i}$. Then the Castelnuovo-Mumford regularity of $I$ is defined as $\operatorname{reg}(I)=$ $\max \left\{e_{i}-i \mid i=0, \ldots, \ell\right\}$ (see [3, 8]). By a well-known result, if coordinates are generic and the monomial ordering is the reverse lexicographic ordering, then reg $(I)$ is an upper bound for the degree of the Gröbner basis elements. This upper bound is reached if the characteristic of $K$ is zero (see [3]).

It follows from these remarks and other similar properties that all computations are much easier if the coordinates are sufficiently generic in order that the above relations are satisfied. More specifically, it is easy to compute the CastelnuovoMumford regularity from a Gröbner basis if the coordinates are sufficiently generic and if one knows that they are. Therefore, one needs a testable definition of sufficiently generic. This definition has to be as large as possible in order to avoid as much as possible a costly change of coordinates.

Received by the editor April 7, 2008 and, in revised form, January 4, 2011.

2010 Mathematics Subject Classification. Primary 14Q20, 13P10.

Key words and phrases. Castelnuovo-Mumford regularity, satiety, degree reverse lexicographic ordering, Gröbner bases, complexity.

(C)2011 American Mathematical Society Reverts to public domain 28 years from publication 
Bayer and Stillman in [3] have already introduced an explicit definition of generic coordinates for a homogeneous ideal. Bermejo and Gimenez in [5] have defined the nice notion of nested type (with a test) for a monomial ideal. But, all of these definitions of generic coordinates are not sufficiently generic such that the degree of the Gröbner basis elements is equal to $\operatorname{reg}(I)$. On the other hand, if $I$ is a Borel monomial ideal and the characteristic of $K$ is zero, then $\operatorname{reg}(I)$ is the maximal degree of the elements of the reduced Gröbner basis of the ideal with respect to the reverse lexicographic ordering (see [2, 3, 7, 10]).

In this paper, we introduce the notion of a homogeneous ideal in quasi stable position (QSP); a new definition for the notion of generic coordinates for computing efficiently the Castelnuovo-Mumford regularity of a homogeneous ideal. This definition is simple to check, because it is tested on the initial ideal for the degree reverse lexicographic ordering. It is explicit, because we provide an algorithm to decide whether a monomial ideal is in QSP or not within a quadratic complexity in input size. We show that, (if the base field is large enough) almost all linear change of variables transform an ideal into QSP. We prove that the Castelnuovo-Mumford regularity of an ideal in QSP is the maximal degree of the elements of its reduced Gröbner basis with respect to the reverse lexicographic ordering. Thus, QSP is a generalization of Borel.

From the computational point of view, there are three important ways to compute the Castelnuovo-Mumford regularity of an ideal.

- Computing the minimal free resolution which is infeasible in practice.

- Making a random linear change of variables in $K^{n(n+1) / 2}$ to put the input ideal in Borel position and computing the Gröbner basis of the changed ideal for testing this position and reading the regularity (3, 10 ).

- Making a random linear change of variables in $K^{n d-d(d-1) / 2}$ where $d$ is the dimension of input ideal to put the input ideal in nested type position, computing the Gröbner basis of the changed ideal to test nested type and then computing the satieties of some monomial ideals associated to this ideal for computing the regularity ([5]).

Roughly speaking, QSP allows for having a method (to compute the regularity of an ideal) with the advantages of the second and third approach, making an incremental random linear change of variables in $K^{N}$ with $N=n(n+1) / 2-(n-d-1)(n-d) / 2$ (which is smaller than $K^{n(n+1) / 2}$ ) then computing the Gröbner basis of the changed ideal for testing QSP (which is less expensive than Borel test and nested type test) and reading the regularity without further computation.

We have implemented an algorithm in the distributed library noether.lib [1] of Singular 13 based on the above results to compute the Castelnuovo-Mumford regularity of an ideal. It makes an incremental (one variable after the other) random linear change of variables to put the input ideal in QSP. We compare its efficiency with the algorithm regIdeal (from library mregular.lib) of SINGULAR via some examples.

The main results of this paper are the following, in which in $(I)$ is the initial ideal of $I$, i.e., the ideal generated by the leading terms of the elements of $I$ with respect to the reverse lexicographic ordering $\prec$ with $x_{n} \prec x_{n-1} \prec \cdots \prec x_{0}$.

- QSP can be tested on the monomial ideal in $(I)$ instead of $I$.

- An algorithm (with a polynomial complexity) for determining whether a monomial ideal is in QSP is given. 
- If the characteristic of $K$ is zero, or sufficiently large, almost all linear changes of variables transform a homogeneous ideal in QSP.

- If $I$ is in QSP, then $\operatorname{reg}(I)=\operatorname{reg}(\operatorname{in}(I))$.

- If $I$ is in QSP, then reg $(I)$ is the maximal degree of the elements of in $(I)$.

- If the characteristic of $K$ is zero, or sufficiently large, one can compute efficiently the regularity of an ideal by putting it in QSP.

Now, we give the structure of the paper. In Sections 2] we describe an algorithm to compute the satiety of an ideal. In Section 3, we define the notion of a homogeneous ideal in QSP. In Section 4, we prove our main result on the Castelnuovo-Mumford regularity of an ideal in QSP, and we describe also an algorithm to compute the Castelnuovo-Mumford regularity of a homogeneous ideal. In Section 5, we show the performance of our algorithms with the existing algorithms of Singular via some examples. Section 6 presents our conclusions.

\section{SATIETy OF AN IDEAL}

In this section we first introduce a new notion of generic coordinates (sat-generic position) to compute the satiety of an ideal. Then, we describe an algorithm to compute the satiety of a general ideal.

We recall first the definition of the saturation and the satiety of a homogeneous ideal. Let $I$ be a homogeneous ideal of the polynomial $\operatorname{ring} R=K\left[x_{0}, \ldots, x_{n}\right]$ where $K$ is an arbitrary field. If $\mathfrak{m}=\left\langle x_{0}, \ldots, x_{n}\right\rangle$ is the unique maximal homogeneous ideal of $R$, then the ideal $I: \mathfrak{m}^{i}$ is defined for any positive integer $i$ as

$$
I: \mathfrak{m}^{i}=\left\{F \in R \mid \forall G \in \mathfrak{m}^{i}, G F \in I\right\} .
$$

The ideal $I: \mathfrak{m}^{\infty}$ is defined $\bigcup_{i=1}^{\infty} I: \mathfrak{m}^{i}$. Denote by $I_{\ell}$ the set of homogeneous elements of degree $\ell$ of $I$.

Proposition 2.1 (Definition). The ideal $I^{\mathrm{sat}}=I: \mathfrak{m}^{\infty}$ is called the saturation of $I$ and it is equal to the unique largest ideal $J \subset R$ having the following property:

$$
\exists m \text { s.t. } \forall \ell \geq m \quad J_{\ell}=I_{\ell} .
$$

Proof. One can see easily that the saturation of $I$ satisfies this property.

Definition 2.1. The satiety of $I$, denoted by sat $(I)$, is the smallest positive integer $m$ such that $I_{\ell}=I_{\ell}^{\text {sat }}$ for all $\ell \geq m$.

Now, we show that if the last variable is generic for an ideal, then its satiety is the same as that of its initial ideal with respect to the reverse lexicographical ordering. For this, we need the following lemmas from [3] in which a linear form $y \in R$ is generic for a homogeneous ideal $I \subset R$ if $y$ is a nonzero divisor in $R / I^{\text {sat }}$.

Lemma 2.1. Let $I \subset R$ be a homogeneous ideal and let $y \in R$ be a linear form. Then the following conditions are equivalent:

(1) $(I: y)_{\ell}=I_{\ell}$ for any $\ell \geq m$.

(2) $\operatorname{sat}(I) \leq m$ and $y$ is generic for $I$.

Lemma 2.2. Let $I \subset R$ be a homogeneous ideal and $m$ a natural number. Then

$$
\left(I: x_{n}\right)_{m}=I_{m} \Leftrightarrow\left(\operatorname{in}(I): x_{n}\right)_{m}=\operatorname{in}(I)_{m} .
$$

Proposition 2.2. Let $x_{n}$ be generic for a homogeneous ideal $I \subset R$. Then $\operatorname{sat}(I)=$ sat $(\operatorname{in}(I))$. 
Proof. We have $\left(I: x_{n}\right)_{\ell}=I_{\ell}$ for any $\ell \geq \operatorname{sat}(I)$ by Lemma 2.1. Thus, (in $(I)$ : $\left.x_{n}\right)_{\ell}=\operatorname{in}(I)_{\ell}$ for any $\ell \geq \operatorname{sat}(I)$ by Lemma 2.2, and this implies that $\operatorname{sat}(\operatorname{in}(I)) \leq$ sat $(I)$ using Lemma 2.1. To prove the other inequality, the same proof works using the fact that $x_{n}$ is also generic for in $(I)$ by Lemma 2.2 .

The following corollary was inspired by [5], Proposition 2.1.

Corollary 2.1. Let $x_{n}$ be generic for a homogeneous ideal $I \subset R$. Then

$$
\operatorname{sat}(I)=\max _{m \in\left(\operatorname{in}(I): x_{n}\right) \backslash \operatorname{in}(I)}\{\operatorname{deg}(m)\}+1 .
$$

Proof. We have sat $(I)$ is equal to sat(in $(I))$ by Proposition 2.2. On the other hand, from hypothesis $x_{n}$ is also generic for $\operatorname{in}(I)$ (Lemma 2.2). Thus, $\left(\operatorname{in}(I): x_{n}\right)_{\ell}=$ in $(I)_{\ell}$ for any $\ell \geq \operatorname{sat}(\operatorname{in}(I))$ by Lemma 2.1. and the satiety of in $(I)$ is the smallest degree from which $\operatorname{in}(I): x_{n}$ and $\operatorname{in}(I)$ are equal. This is equal to the maximum degree of the elements of $\left(\operatorname{in}(I): x_{n}\right) \backslash \operatorname{in}(I)$ plus one which proves the assertion.

We introduce now a new notion to check whether the last variable is sufficiently generic for an ideal to compute its satiety. A homogeneous ideal $I$ is in sat-generic position if the quotient of in $(I)$ with respect to the maximal homogeneous ideal is equal to its quotient with respect to the last variable. The following corollary results easily from Proposition 2.2 and Corollary 2.1

Corollary 2.2. Let $I \subset R$ be a homogeneous ideal in sat-generic position. Then $\operatorname{sat}(I)=\operatorname{sat}(\operatorname{in}(I))$ and this is equal to the maximal degree of the elements of $\operatorname{in}(I)$ containing $x_{n}$.

We describe also an algorithm to check this definition for a monomial ideal.

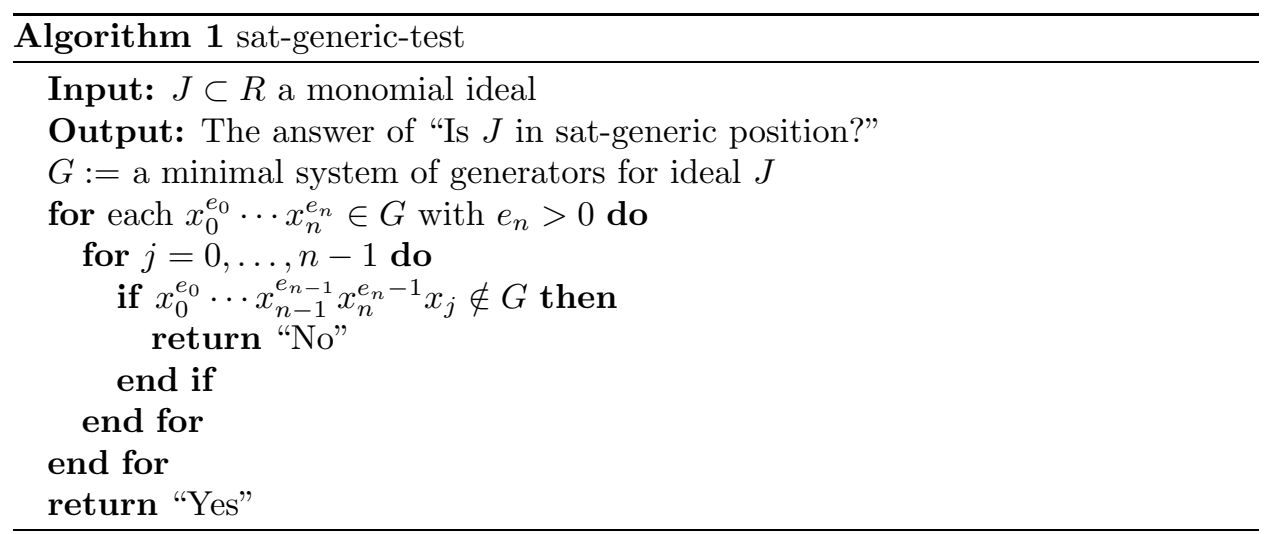

Remark 2.1. The complexity of this algorithm is quadratic in input size.

In the rest of this section, we suppose that the characteristic of $K$ is zero, or sufficiently large. We will prove that almost all linear changes of the last variable transform a homogeneous ideal into sat-generic position. For this we recall some preliminaries from [7, page 352. Let $R_{m}$ be the $K$-vector space consisting of all forms of degree $m$. If $V$ is a $t$-dimensional subspace of of $R_{m}$ and $f_{1}, \ldots, f_{t}$ is a $K$ basis of $V$, then the $t$-exterior power $\wedge^{t} V$ of $V$ is equal to $K f$ where $f=f_{1} \wedge \cdots \wedge f_{t}$. If $f_{1}, \ldots, f_{t}$ and $g_{1}, \ldots, g_{t}$ are linearly independent systems in $R^{m}$, then generate 
the same $K$-subspace iff $f_{1} \wedge \cdots \wedge f_{t}=\lambda g_{1} \wedge \cdots \wedge g_{t}$ for some nonzero $\lambda \in K$. If $m_{1}, \ldots, m_{t}$ are distinct monomials in $R_{m}$, then $m_{1} \wedge \cdots \wedge m_{t}$ is called a monomial of $\wedge^{t} R_{m}$. Such a monomial is called normal expression if $m_{t} \prec \cdots \prec m_{1}$. We order the monomials of $\wedge^{t} R_{m}$ by ordering their normal expressions lexicographically. That is, $m_{1} \wedge \cdots \wedge m_{t}<m_{1}^{\prime} \wedge \cdots \wedge m_{t}^{\prime}$ iff $m_{i} \prec m_{i}^{\prime}$ for the smallest index $i$ such that $m_{i}^{\prime} \neq m_{i}$. Then for each $f \in \wedge^{t} R_{m}$, we can consider its initial monomial in $(f)$.

Let $I \subset R$ be a homogeneous ideal. For all $\Lambda=\left(a_{0}, \ldots, a_{n-1}\right)$ in $K^{n}$, denote by $\Lambda(I)$ the result of the substitution $x_{n} \mapsto x_{n}+a_{n-1} x_{n-1}+\cdots+a_{0} x_{0}$ in $I$.

Recall that the Zariski topology on $K^{n}$ is the topology which has the algebraic sets as closed sets, i.e., a set $V$ is closed if and only if there exists a (finite) family of polynomials $f_{1}, \ldots, f_{k} \in K\left[a_{0}, \ldots, a_{n-1}\right]$ such that

$$
V=\left\{\Lambda \in K^{n} \mid f_{1}(\Lambda)=\cdots=f_{k}(\Lambda)=0\right\} .
$$

It is well known that a nonempty Zariski open set is dense. Under these assumptions, by a restriction to the last variable of Galligo's theorem [10] (see also [7, Theorem 15.18) we have the following theorem.

Theorem 2.1. Let $K$ be an infinite field. Then, there is a monomial ideal $J \subset R$ and a Zariski open set $U \subset K^{n}$ such that $\operatorname{in}(\Lambda(I))=J$ for each $\Lambda \in U$. Moreover, for each $m \geq 0$, if $J_{m}$ has dimension $t, \wedge^{t} J_{m}$ is generated by $\max \{\operatorname{in}(f) \mid f \in$ $\left.\wedge^{t} \Lambda(I)_{m}, \Lambda \in K^{n}\right\}$.

Proof. Let $f_{1}, \ldots, f_{t}$ be a basis for $I_{m}$ and $f=f_{1} \wedge \cdots \wedge f_{t}$. For any $\Lambda=$ $\left(a_{0}, \ldots, a_{n-1}\right)$ we have $\Lambda(f)=\Lambda\left(f_{1}\right) \wedge \cdots \wedge \Lambda\left(f_{t}\right)$ is a linear combination of monomials in $\wedge^{t} R_{m}$. Let $p\left(a_{0}, \ldots, a_{n-1}\right) \subset K\left[a_{0}, \ldots, a_{n-1}\right]$ be the coefficient of $\operatorname{in}(\Lambda(f))$. Now set

$$
U_{m}=\left\{\Lambda \in K^{n} \mid p\left(a_{0}, \ldots, a_{n-1}\right) \neq 0\right\} .
$$

Let $J_{m}$ be the subspace of $R_{m}$ generated by $m_{1}, \ldots, m_{t}$ where $\operatorname{in}(\Lambda(f))=m_{1} \wedge \cdots \wedge$ $m_{t}$. Set $J=\sum_{m \geq 1} J_{m}$ and $U=\bigcap_{m=1}^{\infty} U_{m}$. We now show that $J$ is an ideal and $U$ is a Zariski open set. To show that $J$ is an ideal, it suffices to prove that $R_{i} J_{m} \subset R_{m+i}$ for any $i$ and $m$. Note that $U_{m}$ and $U_{m+i}$ are dense, therefore $U_{m} \cap U_{m+i} \neq \emptyset$. Pick $\Lambda \in U_{m} \cap U_{m+i}$, it follows that $\operatorname{in}(\Lambda(I))_{m}=J_{m}$ and $\operatorname{in}(\Lambda(I))_{m+i}=J_{m+i}$. Since in $(\Lambda(I))$ is an ideal, we can conclude the desired inclusion. Also, the ideal $J$ satisfies the last statement of the theorem.

We will show that $U$ is a Zariski open set of $K^{n}$. Since each $U_{m}$ is Zariski open, it is enough to show that $U$ is equal to finite intersection of the $U_{m}$. Suppose that $J$ is generated by $m_{1}, \ldots, m_{s}$. Let $e$ be the highest degree of these monomials. We claim that $U=\bigcap_{m=1}^{e} U_{m}$. Take $\Lambda \in \bigcap_{m=1}^{e} U_{m}, \operatorname{in}(\Lambda(I))_{m}=J_{m}$ for any $m \leq e$, hence $J \subset \operatorname{in}(\Lambda(I))$. Since $\operatorname{dim}_{K} J_{m}=\operatorname{dim}_{K} I_{m}=\operatorname{dim}_{K} \operatorname{in}(\Lambda(I))_{m}$ for every $m$ we have $J=\operatorname{in}(\Lambda(I))$ and thus $\Lambda \in U$. The other inclusion is trivial. This argument was a rewriting of the proof of [7, Theorem 15.18.

Corollary 2.3. Let the characteristic of $K$ be zero, or sufficiently large. With the notation of Theorem 2.1, $J$ is in sat-generic position (and therefore almost all linear changes of the last variable transform I in sat-generic position).

Proof. We have to show that if $x_{n} m \in J$, then $x_{i} m \in J$. Let $\Lambda_{i}=(0, \ldots, 0,1$, $0, \ldots, 0) \in K^{n}$ such that the $i$ th element is one and the others are zero. So, it is enough to prove that $\Lambda_{i}\left(J_{s}\right)=J_{s}$ with $s=\operatorname{deg}(m)+1$. Also, let $\Lambda$ be an element of $K^{n}$ such that $\operatorname{in}(\Lambda(I))=J$. Choose a basis $f_{1}, \ldots, f_{t}$ for $\Lambda(I)_{s}$. Set $f=f_{1} \wedge \cdots \wedge f_{t}$. Thus $\operatorname{in}(f)=\operatorname{in}\left(f_{1}\right) \wedge \cdots \wedge \operatorname{in}\left(f_{t}\right)$. 
If $\Lambda_{i}\left(J_{s}\right) \neq J_{s}$, then $\Lambda_{i}(\operatorname{in}(f)) \neq \operatorname{in}(f)$. By definition of $\Lambda_{i}(\operatorname{in}(f))$, its terms (other than in $(f)$ ) are strictly greater than in $(f)$. We will show that for a suitable $\Lambda^{\prime} \in K^{n}$, a monomial (with nonzero coefficient) strictly greater than in $(f)$ appears in $\Lambda^{\prime}(f)$. This contradicts the last statement of Theorem 2.1, proving the assertion.

Let $\Lambda^{\prime}$ be the vector $\Lambda_{i}$ by replacing 1 by $c$ where $c$ is a generic element of $K$. Let $f_{i}=c_{i} x_{n}^{p_{i}} f_{i}^{\prime}$ for any $i$ where $p_{i}$ is a nonnegative integer and $f_{i}^{\prime} \in R$ is a polynomial whose leading coefficient is 1 . Let $m$ be the normal expression form of $x_{i}^{p_{1}} \operatorname{in}\left(f_{1}^{\prime}\right) \wedge \cdots \wedge x_{i}^{p_{t}} \operatorname{in}\left(f_{t}^{\prime}\right)$. It is trivial that $m$ appears in $\Lambda^{\prime}(f)$. We claim that $m$ is strictly greater than in $(f)$ and it has a nonzero coefficient if $c \in K$ is sufficiently generic. From supposition and definition of orderings, it is clear that $m$ is strictly greater than in $(f)$. To prove the other part of claim, let $p(c)$ be the coefficient of $m$ in $f$. One can see easily that $c_{1} \cdots c_{t} c^{p_{1}+\cdots+p_{t}}$ is the leading coefficient of $p(c)$ (which is nonzero). By the assumption on the characteristic of $K$, it follows that for a sufficiently generic value of $c$, the value of $p(c)$ is nonzero and this ends the proof. This argument was inspired by the proof of [7, Theorem 15.20.

Example 2.1. If $K$ is finite and its characteristic is not sufficiently large, Corollary 2.3 may be false. For example, let the characteristic of $K$ be two and $I=\left\langle x^{2}, y^{2}\right\rangle \subset$ $K[x, y]$. Then, any linear change of coordinates transforms $I$ to itself which is not in sat-generic position, because $I: y=\left\langle x^{2}, y\right\rangle$ is not equal to $I:\langle x, y\rangle=\left\langle x^{2}, x y, y^{2}\right\rangle$.

Finally, we describe our algorithm to compute the satiety of a general ideal. For this, we denote by $D(n, J)$ the maximal degree of the elements of a minimal system of generators of a monomial ideal $J$ containing $x_{n}$. The correctness and termination of this algorithm follow, respectively, from Corollaries 2.2 and 2.3 We have implemented this algorithm in SINGULAR and we compare its running time with satiety and satiety(.,1) (from the library mregular.lib) of Singular in Section 5.

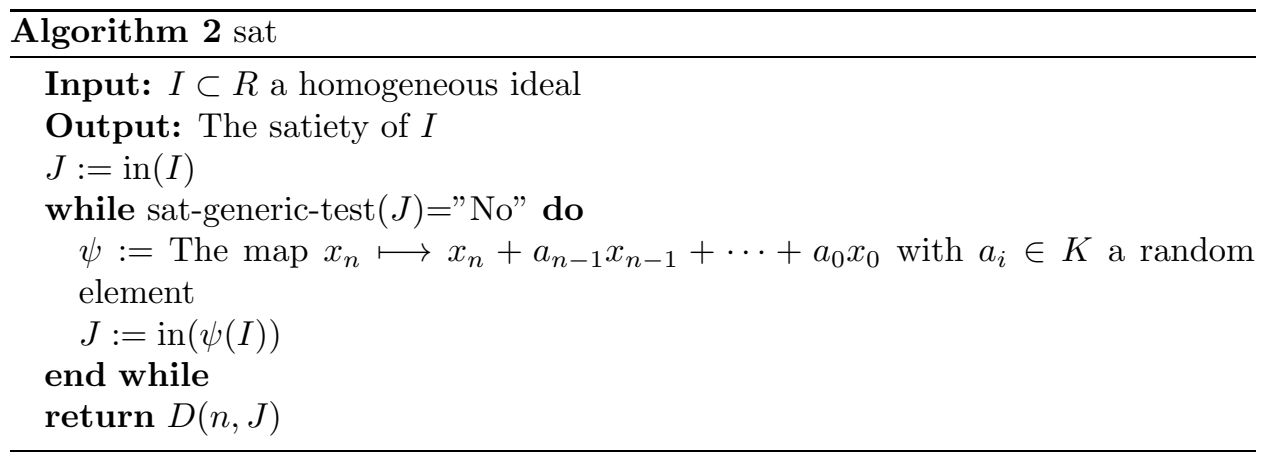

\section{QuAsi STABLE POSITION}

In this section, we define the new notion of a homogeneous ideal in quasi stable position (QSP). We show also the relationship between this notion and the other existing notions of generic coordinates for computing the Castelnuovo-Mumford regularity of a homogeneous ideal.

To define the notion QSP, we need the following sequence of ideals associated to an ideal in which $J$ is a monomial ideal of the ring $R=K\left[x_{0}, \ldots, x_{n}\right]$ and $\mathfrak{m}_{\mathfrak{i}}=\left\langle x_{0}, \ldots, x_{i}\right\rangle$ is the unique maximal ideal of the ring $R_{i}=K\left[x_{0}, \ldots, x_{i}\right]$. 
Notation 3.1. Let $\overline{\sec }(J, 0)=J$ and $\overline{\sec }(J, i)=\left.J\right|_{x_{n-i+1}=\cdots=x_{n}=0} \cap R_{n-i}$ for $i=$ $1, \ldots, n$.

Definition 3.1. A monomial ideal $J$ is in quasi stable position (QSP) if we have $\overline{\sec }(J, i): \mathfrak{m}_{\mathfrak{n}-\mathfrak{i}}=\overline{\sec }(J, i): x_{n-i}$ for $i=0, \ldots, \operatorname{dim}(J)$.

Definition 3.2. A homogeneous ideal $I \subset R$ is in quasi stable position (QSP) if $\operatorname{in}(I)$ is in QSP.

We note an immediate consequence of these definitions.

Corollary 3.1 (QSP-test). Let $J$ be a monomial ideal and $w(m)$ the maximal integer $\ell$ such that $x_{\ell}$ divides a monomial $m$. The following conditions are equivalent:

- $J$ is in QSP.

- For all monomial $m \in J$, if $w(m) \geq n-\operatorname{dim}(J)$ we have $x_{i}\left(m / x_{w(m)}\right) \in J$ for all $i<w(m)$.

Using this test, we give an algorithm to decide whether a monomial ideal is in QSP.

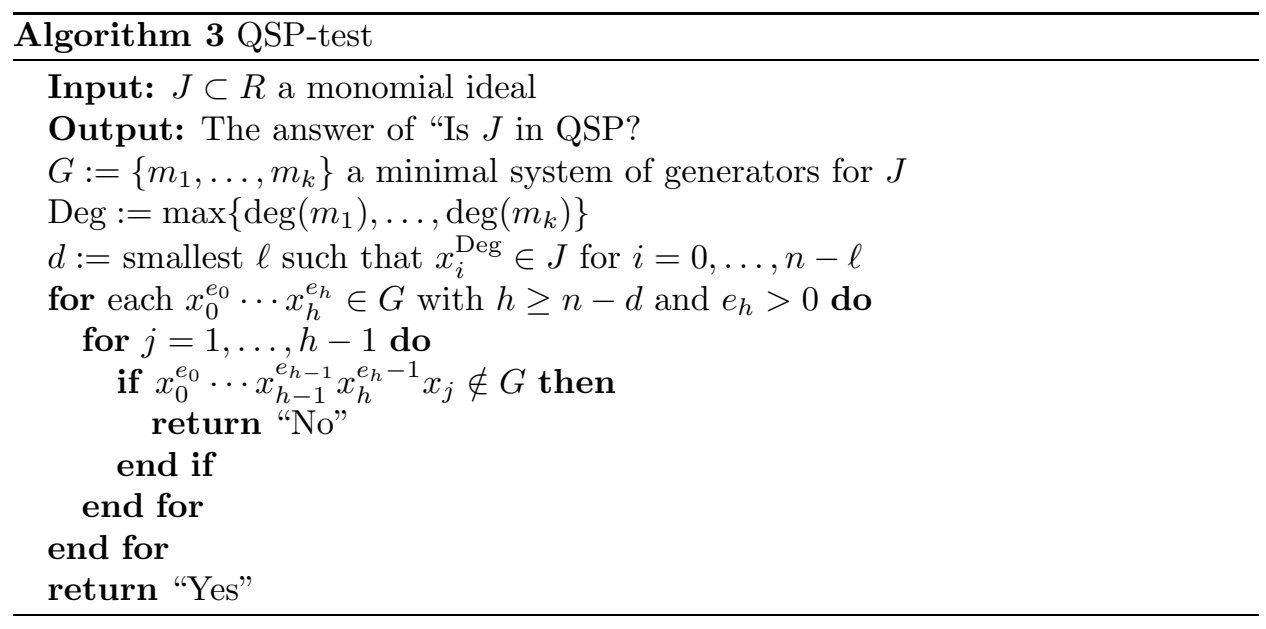

Proof of algorithm QSP-test. The termination of the algorithm is obvious. Let us show its correctness. For this, we have to prove that $J$ is in QSP if and only if the response of the algorithm is "Yes". Suppose that $J$ is in QSP and $x_{0}^{e_{0}} \cdots x_{h}^{e_{h}} \in G$ for some $h \geq n-d$ with $e_{h}>0$. This implies that

$$
x_{0}^{e_{0}} \cdots x_{h-1}^{e_{h-1}} x_{h}^{e_{h}-1} \in J: x_{h}=J:\left\langle x_{0}, \ldots, x_{h}\right\rangle .
$$

Thus, $x_{0}^{e_{0}} \cdots x_{h-1}^{e_{h-1}} x_{j}$ must be in $J$ for any $j=0, \ldots, h-1$, and the answer is "Yes". In this case, $d$ is the dimension of $J$ by the Noether normalization test (see [4, Lemma 3.1). Conversely, we can conclude that $J: x_{h} \subset J:\left\langle x_{0}, \ldots, x_{h}\right\rangle$, and therefore $J$ is in QSP by definition of the notation.

Remark 3.1. In this algorithm, the integer $d$ is the dimension of $J$ if the answer is "Yes" (see the proof of algorithm).

Proposition 3.1. The complexity of this algorithm is quadratic in $k n$. 


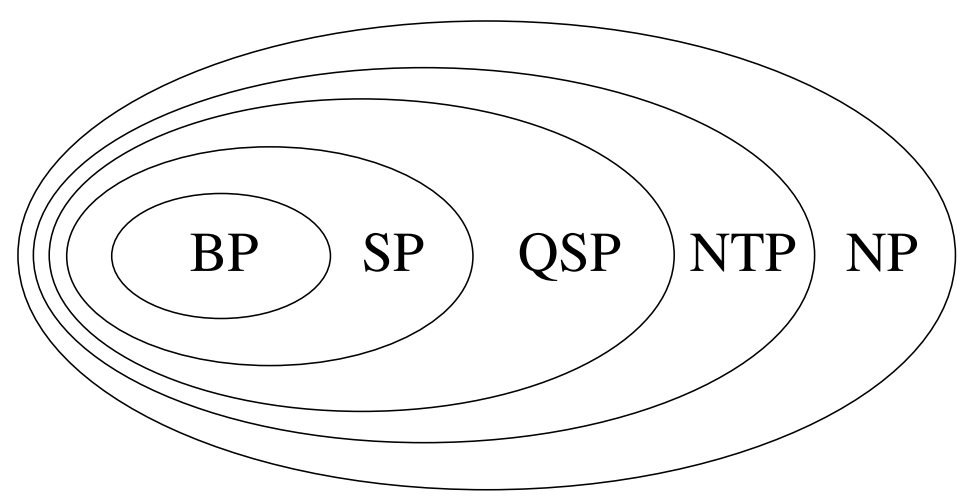

Figure 1. Classes of ideals

Proof. One can easily see that the number of operations in the loop "For" is $k n^{2}$. Thus the complexity of the algorithm is polynomial in $k n$.

Example 3.1. There is a nontrivial homogeneous ideal in QSP. Set $R=\mathbb{Q}[x, y, z$, $t, u, v, h]$. Let $I$ be the homogenization of the 5 -th Katsura ideal with respect to $h$, i.e., the homogenization of the ideal generated by $2 x^{2}+2 y^{2}+2 z^{2}+2 t^{2}+2 u^{2}+v^{2}-$ $v, x y+y z+2 z t+2 t u+2 u v-u, 2 x z+2 y t+2 z u+u^{2}+2 t v-t, 2 x t+2 y u+2 t u+2 z v-z, t^{2}+$ $2 x v+2 y v+2 z v-y$ and $2 x+2 y+2 z+2 t+2 u+v-1$. Its dimension is 1 , and its initial ideal, in $(I)$, is generated by $x, t^{2}, z t, y t, y z, y^{2}, u^{3}, t u^{2}, z u^{2}, y u^{2}, z^{2} u, z^{3}, u^{2} v^{2}, t u v^{2}$, $z u v^{2}, y u v^{2}, z^{2} v^{2}, u v^{4}, t v^{4}, z v^{4}, y v^{4}, v^{6}$. One can easily check that this initial ideal is in QSP by the above algorithm, and therefore I is in QSP by definition.

We now compare QSP with some other notions of generic coordinates. Recall that a monomial ideal $J \subset R$ is in nested type position $(\mathrm{NTP})$ if $\overline{\sec }(J, i)^{\mathrm{sat}}=$ $\overline{\sec }(J, i): x_{n-i}^{\infty}$ for $i=0, \ldots, \operatorname{dim}(J)-1$ (see [5], Proposition 3.2).

Lemma 3.1. Any monomial ideal in QSP is in NTP.

Proof. Let $J$ be a monomial ideal in QSP. We have to show that any monomial $m \in \overline{\sec }(\operatorname{in}(J), i): x_{n-i}^{\infty}$ belongs to $\overline{\sec }(\operatorname{in}(J), i)^{\text {sat }}$ for any $i<\operatorname{dim}(J)$. Let $x_{n-i}^{k} m \in$ $\overline{\sec }(\operatorname{in}(J), i)$ for some integers $k$ and $i$. From hypothesis we have $x_{j}^{k} m \in \overline{\sec }(\operatorname{in}(J), i)$ for any $j<n-i$, and this follows the assertion.

Recall that a monomial ideal $J \subset R$ is Borel if it is fixed under the action of the upper triangular invertible matrices in $G L(n+1, K)$. Provided the characteristic of $K$ is zero, a monomial ideal $J \subset R$ is Borel if and only if $x_{j} m \in J$ implies that $x_{i} m \in J$ for all $i<j$. Also, there is another class of monomial ideals called stable ideals (see [9]). A monomial ideal $J \subset R$ is stable if the equality of Definition 3.1 is true for $i=0, \ldots, n-1$. Thus, in characteristic zero a Borel monomial ideal is a stable ideal, and in any characteristic a stable ideal is in QSP. These imply that QSP is a notion between stable and NTP. In Figure 1, we compare the different notions of generic coordinates in which BP (resp. SP, NTP and NP) shows the class of the ideals whose initial ideals are in Borel position (resp. stable position, NTP and Noether position). Recall that a monomial ideal $J$ is in Noether position if a pure power of $x_{i}$ belongs to $J$ for $i=0, \ldots, n-\operatorname{dim}(J)$ (see [4]). Note that QSP and NTP are not equivalent. For example, the ideal $\left\langle x^{2}, y^{2}\right\rangle \subset \mathbb{Q}[x, y]$ is in NTP, 
but it is not in QSP. Also, QSP is not equivalent to SP, because, for example, the initial ideal of Example 3.1 is an ideal in QSP but it is not in SP (this is also true for the initial ideal of the 6, 7, 8, 9 and 10-th Katsura ideals).

In the rest of this section we suppose that the characteristic of $K$ is zero, or sufficiently large. We show that for almost all linear change of variables an ideal is in QSP. Let $I \subset R$ be a homogeneous ideal of dimension $d$. For all $\Lambda=$ $\left(a_{1}, \ldots, a_{N}\right) \in K^{N}$ with $N=n(n+1) / 2-(n-d-1)(n-d) / 2$, denote by $\Lambda(I)$ the result of the following substitution in $I$ :

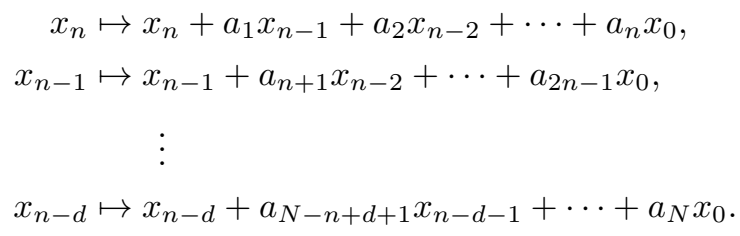

Under these assumptions, we can conclude the following.

Corollary 3.2. Let the characteristic of $K$ be zero, or sufficiently large. There is a monomial ideal $J \subset R$ and a Zariski open set $U \subset K^{N}$ such that $\operatorname{in}(\Lambda(I))=J$ for each $\Lambda \in U$. Moreover, $J$ is in QSP (and thus almost all linear changes of the variables $x_{n}, \ldots, x_{n-d}$ put $I$ in QSP).

Proof. The assertion follows by applying Corollary 2.3 to the variables $x_{n}, \ldots, x_{n-d}$.

For finite fields, this is not always true. For instance, in characteristic $p>0$, there is no linear change of variables to put the ideal $\left\langle x^{p}, y^{p}\right\rangle$ in QSP. Note that the fact that the characteristic of $K$ is small causes this problem.

\section{Castelnuovo-Mumford Regularity of an ideal in QSP}

In this section, we prove the main result of this paper: the Castelnuovo-Mumford regularity of an ideal in QSP is the maximal degree of the elements of its reduced Gröbner basis with respect to the reverse lexicographic ordering. We describe also an algorithm based on this result to compute the Castelnuovo-Mumford regularity of a general homogeneous ideal.

We prove first that the maximal degree of the elements of the reduced Gröbner basis of an ideal in QSP is less than or equal to its Castelnuovo-Mumford regularity (Proposition 4.2).

Lemma 4.1 ([3]). Let $I \subset R$ be a homogeneous ideal, and $y \in R$ a generic linear form for $I$. The following conditions are equivalent:

- $\operatorname{reg}(I) \leq m$.

- $\operatorname{sat}(I) \leq m$ and $\operatorname{reg}(I+\langle y\rangle) \leq m$.

Lemma 4.2 ([3]). Let $I \subset R$ be a zero-dimensional homogeneous ideal. The following conditions are equivalent:

- $\operatorname{sat}(I) \leq m$.

- $\operatorname{reg}(I) \leq m$.

- $I_{m}=R_{m}$.

Corollary 4.1. With the same hypothesis $\operatorname{reg}(I)=\operatorname{sat}(I)$. 
Lemma 4.3 ([12]). Let $I \subset R$ homogeneous ideal. Then

$$
\operatorname{reg}(I)=\max \left\{\operatorname{sat}(I), \operatorname{reg}\left(I^{\mathrm{sat}}\right)\right\} .
$$

We need some more notation as follows: We define $J \subset R$ to be a monomial ideal. Let $\sec (J, 0)=J$ and $\sec (J, i)=J+\left\langle x_{n-i+1}, \ldots, x_{n}\right\rangle$ for $i=1, \ldots, n$.

Proposition 4.1. Let $J \subset R$ be a monomial ideal in QSP and let $d=\operatorname{dim}(J)$, then

$$
\begin{aligned}
\operatorname{reg}(J) & =\max _{0 \leq i \leq d}\{\operatorname{sat}(\sec (J, i))\} \\
& =\max _{0 \leq i \leq d}\{\operatorname{sat}(\overline{\sec }(J, i))\} .
\end{aligned}
$$

Proof. To prove the equality (1), from Lemma 4.1 we have

$$
\operatorname{reg}(J)=\max \{\operatorname{sat}(J), \operatorname{reg}(\sec (J, 1))\} .
$$

By reusing this formula for the ideal $\sec (J, 1)$ and using the fact that $x_{n-1}$ is generic for $\sec (J, 1)$ we obtain

$$
\begin{aligned}
\operatorname{reg}(J) & =\max \{\operatorname{sat}(J), \max \{\operatorname{sat}(\sec (J, 1)), \operatorname{reg}(\sec (J, 2))\}\} \\
& =\max \{\operatorname{sat}(J), \operatorname{sat}(\sec (J, 1)), \operatorname{reg}(\sec (J, 2))\}
\end{aligned}
$$

So by an induction, we can conclude that $\operatorname{reg}(J)$ is equal to

$$
\max \{\operatorname{sat}(J), \operatorname{sat}(\sec (J, 1)), \ldots, \operatorname{sat}(\sec (J, d-1)), \operatorname{reg}(\sec (J, d))\} \text {. }
$$

Since $\sec (J, d)$ is zero-dimensional, then $\operatorname{reg}(\sec (J, d))=\operatorname{sat}(\sec (J, d))$ (Corollary 4.1), and this proves the assertion.

Let us prove (2). It is enough to show that $\operatorname{sat}(\overline{\sec }(J, i))=\operatorname{sat}(\sec (J, i))$ for any $i$. By the membership $x_{n-i+1}, \ldots, x_{n} \in \sec (J, i)$ we have

$$
\overline{\sec }(J, i)^{\mathrm{sat}}=\left.\sec (J, i)^{\mathrm{sat}}\right|_{x_{n-i+1}=\cdots=x_{n}=0} \cap R_{n-i}
$$

by definition of the saturation of an ideal, and this proves the assertion.

Lemma 4.4. If $I \subset R$ is a homogeneous ideal in QSP then $\operatorname{reg}(I)=\operatorname{reg}(\operatorname{in}(I))$.

Proof. Following [3], a sequence $y_{1}, \ldots, y_{i} \in R$ of linear forms is called generic for an ideal $I \subset R$ if $y_{j}$ is generic for $I+\left\langle y_{1}, \ldots, y_{j-1}\right\rangle$ for any $j=1, \ldots, i$. By hypothesis the sequence $x_{n}, \ldots, x_{n-\operatorname{dim}(I)+1}$ is generic for in $(I)$. Thus the assertion follows from [3], Theorem 2.4.

Proposition 4.2. Let $I \subset R$ be a homogeneous ideal in QSP. The maximal degree of the elements of the reduced Gröbner basis of I is less than or equal to reg $(I)$.

Proof. Using Lemma 4.4, it is enough to prove the assertion for $J=\operatorname{in}(I)$ which is a monomial ideal. Let us consider $G=\left\{m_{1}, \ldots, m_{s}\right\}$ a minimal system of generators of $J$ and a monomial $m_{j}$ in $G$. Let $x_{i}$ be the greatest variable which divides $m_{j}$. Two cases are possible: If $i \in\{n-d+1, \ldots, n\}$ with $d=\operatorname{dim}(J)$, these assumptions imply that $m_{j} / x_{i} \in \overline{\sec }(J, n-i): x_{i}$ and $m_{j} / x_{i} \notin \overline{\sec }(J, n-i)$. In addition, $\overline{\sec }(J, n-i)^{\mathrm{sat}}=\overline{\sec }(J, n-i): x_{i}^{\infty}$ because $J$ is in QSP. This implies that $m_{j} / x_{i} \in \overline{\sec }(J, n-i)^{\mathrm{sat}}$. The facts that $m_{j} / x_{i} \in \overline{\sec }(J, n-i)^{\mathrm{sat}}$ and $m_{j} / x_{i} \notin$ $\overline{\sec }(J, n-i)$ result in $\operatorname{deg}\left(m_{j}\right) \leq \operatorname{sat}(\sec (J, n-i))$ by definition. Thus $\operatorname{deg}\left(m_{j}\right)$ is less than or equal to $\operatorname{reg}(J)$ by Proposition 4.1.

In the other case, i.e., $i \in\{0, \ldots, n-d\}$, we use the facts that $\sec (J, d)$ is zerodimensional and its regularity is less than or equal to $\operatorname{reg}(J)$ (Lemma 4.1 and the 
fact that $J$ is in QSP). Suppose ad absurdum that $\operatorname{deg}\left(m_{j}\right)>\operatorname{reg}(J)$ for some $j$. Let $m$ be a monomial of degree $\operatorname{reg}(J)$ which divides $m_{j}$. Thus $m \in \overline{\sec }(J, d)$ by Lemma 4.2, and $m \in J$ by supposition. This is a contradiction with minimality of $G$ which follows that $\operatorname{deg}\left(m_{j}\right) \leq \operatorname{reg}(J)$, and this proves the assertion.

Bayer and Stillman 3. have proved that if the characteristic of $K$ is zero, then the Castelnuovo-Mumford regularity of a Borel monomial ideal is the maximal degree of the elements of its reduced Gröbner basis with respect to the reverse lexicographic ordering. In the following, we generalize this result to any characteristic and the ideals in QSP.

Theorem 4.1. The Castelnuovo-Mumford regularity of an ideal in QSP is the maximal degree of the elements of its reduced Gröbner basis with respect to the reverse lexicographic ordering.

Proof. Let $I \subset R$ be an ideal in QSP. Also, let $D$ be the maximal degree of the elements of $\operatorname{in}(I)$. From Proposition 4.2, we have $D \leq \operatorname{reg}(I)$. To prove the other inequality, from Proposition 4.1 (2) and Lemma 4.4 we have $\operatorname{reg}(I)=\operatorname{reg}(\operatorname{in}(I)) \leq$ $\operatorname{sat}(\overline{\sec }(\operatorname{in}(I), i))$ for some $i$ with $0 \leq i \leq \operatorname{dim}(I)$. From hypothesis, $\overline{\sec }(\operatorname{in}(I), i)$ is in QSP, and its satiety is equal to the maximal degree of the elements of in $(I)$ containing $x_{n-i}$ by Corollary 2.2 . Thus, $\operatorname{reg}(I) \leq D$.

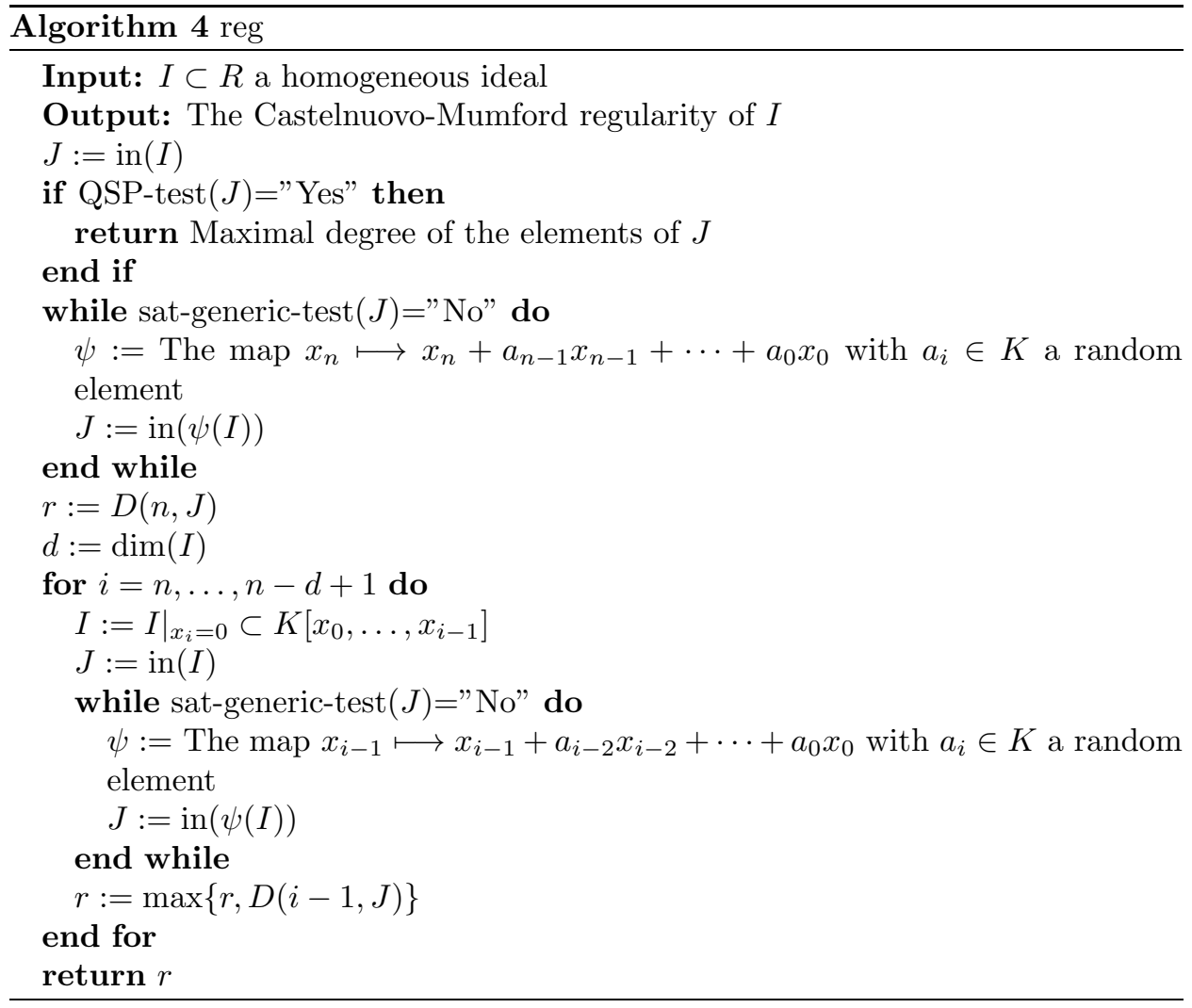

This algorithm is based on the above theorem for computing the CastelnuovoMumford regularity of a general ideal by an incremental (one variable after the 
other) random linear change of variables to put the input ideal in QSP. We denote by $D(i, J)$ the maximal degree of the elements of a minimal system of generators of a monomial ideal $J$ containing $x_{i}$. The correctness and termination of this algorithm follow, respectively, from Theorem 4.1 and Corollary 3.2. We have implemented this algorithm in the distributed library noether.lib [1] of Singular and we compare its running time with the function regIdeal (from the library mregular.lin) of Singular in Section 5 .

\section{EXPERIMENTS AND REMARKS}

We have implemented sat and reg algorithms with the computer algebra system Singular (version 3-0-1). We have also implemented the msat (resp. mreg) algorithm which is a modular version of sat (resp. reg) algorithm, i.e., it computes the initial ideal modulo 2147483647 (the biggest prime number less than $2^{31}$ ) with a high probability. All of these algorithms are contained in the distributed library noether.lib 1 .

We choose Singular since it has many functionalities needed which are related to a multivariate polynomial ring. Also, we want to be able to compare our algorithms with the existing functions: satiety, satiety $(., 1)$ and regIdeal of Singular.

For this experiment, we use some examples from [6], the Posso list1 and [5]. For the polynomial systems which are not homogeneous, we use their homogenization. The results are shown in Table 1 . All of the computations are done over $\mathbb{Q}$. The monomial ordering is always degree reverse lexicographical ordering. In this table, Example 1 is the ideal of the ring $\mathbb{Q}[x, y, z]$ generated by $8 x^{2} y^{2}+5 x y^{3}+3 x^{3} z+$ $x^{2} y z, x^{5}+2 y^{3} z^{2}+13 y^{2} z^{3}+5 y z^{4}, 8 x^{3}+12 y^{3}+x z^{2}, 7 x^{2} y^{4}+18 x y^{3} z^{2}+y^{3} z^{3}$. Examples $2,3,5,8,9,10,12,14,15,16,17$ and 18 are, respectively, Siebert, Macaulay, Schwarz, Cassou, Horrocks, Huneke, Amrhein2, Gerdt, Möller, Sturmfels/Eisenbud, mat $3^{2}$ and Butcher Examples (see [6), and Examples 4,6,7 and 11 are, respectively, Bronstein2, Cyclic roots 5, Katsura4 and Katsura5 Examples from the Posso list. Examples 13 and 20 are respectively Examples 4.13 and 4.10 from [5]. Example 19 is Shimoyama/Yokoyama example and it is the ideal generated by the polynomials $-j^{9}+9 j^{8} a-36 j^{7} a^{2}+84 j^{6} a^{3}-126 j^{5} a^{4}+126 j^{4} a^{5}-84 j^{3} a^{6}+36 j^{2} a^{7}-9 j a^{8}+$ $a^{9},-b j^{8}+8 b j^{7} a+j^{8} a-28 b j^{6} a^{2}-8 j^{7} a^{2}+56 b j^{5} a^{3}+28 j^{6} a^{3}-70 b j^{4} a^{4}-56 j^{5} a^{4}+$ $56 b j^{3} a^{5}+70 j^{4} a^{5}-28 b j^{2} a^{6}-56 j^{3} a^{6}+8 b j a^{7}+28 j^{2} a^{7}-b a^{8}-8 j a^{8}+a^{9}, c j^{7}-7 c j^{6} a-$ $j^{7} a+21 c j^{5} a^{2}+7 j^{6} a^{2}-35 c j^{4} a^{3}-21 j^{5} a^{3}+35 c j^{3} a^{4}+35 j^{4} a^{4}-21 c j^{2} a^{5}-35 j^{3} a^{5}+$ $7 c j a^{6}+21 j^{2} a^{6}-c a^{7}-7 j a^{7}+a^{8},-d j^{6}+6 d j^{5} a+j^{6} a-15 d j^{4} a^{2}-6 j^{5} a^{2}+20 d j^{3} a^{3}+$ $15 j^{4} a^{3}-15 d j^{2} a^{4}-20 j^{3} a^{4}+6 d j a^{5}+15 j^{2} a^{5}-d a^{6}-6 j a^{6}+a^{7}, e j^{5}-5 e j^{4} a-j^{5} a+$ $10 e j^{3} a^{2}+5 j^{4} a^{2}-10 e j^{2} a^{3}-10 j^{3} a^{3}+5 e j a^{4}+10 j^{2} a^{4}-e a^{5}-5 j a^{5}+a^{6},-f j^{4}+$ $4 f j^{3} a+j^{4} a-6 f j^{2} a^{2}-4 j^{3} a^{2}+4 f j a^{3}+6 j^{2} a^{3}-f a^{4}-4 j a^{4}+a^{5}, g j^{3}-3 g j^{2} a-j^{3} a+$ $3 g j a^{2}+3 j^{2} a^{2}-g a^{3}-3 j a^{3}+a^{4},-h j^{2}+2 h j a+j^{2} a-h a^{2}-2 j a^{2}+a^{3}, i j-i a-j a+a^{2}$ in the ring $\mathbb{Q}[a, b, c, d, e, f, g, h, i, j]$.

In Table 1, the column var (resp. dim, sat, reg and ncv) shows the number of variables of the ring (resp. the dimension, the satiety, the Castelnuovo-Mumford regularity of the ideal and the number of variables which are needed to change to put the ideal in QSP). The other columns show the timing of computing by the corresponding algorithm in which timing is measured in seconds (with a precision of hundredths of a second) and the symbol $\infty$ means more than 24 hours. The

\footnotetext{
${ }^{1}$ http://www-sop.inria.fr/saga/POL/BASE/3.posso/
} 
TABLE 1. Comparison of msat, sat, satiety and satiety $(., 1)$ and of mreg, reg and regIdeal algorithms

\begin{tabular}{|c||c|c|c|c|c|c|c|c|c|c|c|c|}
\hline Example & var & dim & sat & msat & sat & satiety & satiety (., 1) & reg & ncv & mreg & reg & regIdeal \\
\hline \hline 1 & 3 & 1 & 8 & 0.01 & 0.01 & 0.06 & 0.01 & 8 & 0 & 0.01 & 0.01 & 0.01 \\
2 & 5 & 2 & 9 & 0.03 & 0.11 & 0.06 & 8.62 & 9 & 2 & 0.06 & $* 0.80$ & 11.79 \\
3 & 5 & 3 & 0 & 0.00 & 0.00 & 0.01 & 0.43 & 7 & 2 & 0.02 & 0.02 & 0.43 \\
4 & 5 & 3 & 0 & 0.00 & 0.00 & 0.01 & 0.04 & 6 & 2 & 0.02 & 0.03 & 0.05 \\
5 & 6 & 1 & 0 & 0.01 & 0.26 & 0.01 & 0.18 & 6 & 1 & 0.02 & 0.15 & 0.32 \\
6 & 6 & 1 & 0 & 0.03 & 5.50 & 0.21 & 5.80 & 11 & 1 & 0.06 & 3.96 & 5.98 \\
7 & 6 & 1 & 0 & 0.00 & 0.00 & 0.15 & 0.01 & 5 & 0 & 0.01 & 0.01 & 0.01 \\
8 & 6 & 2 & 0 & 0.43 & 11.17 & $* 0.37$ & $\infty$ & 16 & 3 & 1.58 & $* 2420.72$ & $\infty$ \\
9 & 6 & 3 & 0 & 0.03 & 0.11 & 0.02 & 1.16 & 5 & 3 & 0.11 & 0.34 & 1.28 \\
10 & 6 & 3 & 0 & 0.02 & 0.03 & 0.66 & $\infty$ & 42 & 3 & 7.98 & $\infty$ & $\infty$ \\
11 & 7 & 1 & 0 & 0.01 & $* 0.05$ & 4.59 & 0.06 & 6 & 0 & 0.02 & 0.06 & 0.07 \\
12 & 8 & 1 & 0 & 0.01 & $* 0.01$ & 0.45 & 0.02 & 8 & 0 & 0.04 & 0.04 & 0.05 \\
13 & 8 & 2 & 0 & 0.09 & 0.12 & 2.57 & 49.23 & 6 & 1 & 0.27 & $* 3.14$ & 46.07 \\
14 & 8 & 3 & 7 & 0.08 & 0.32 & 0.23 & 1645.79 & 7 & 4 & 0.36 & $* 32.32$ & 1610.60 \\
15 & 8 & 3 & 7 & 0.04 & 0.26 & 0.06 & 6.44 & 7 & 2 & 0.07 & 0.32 & 8.96 \\
16 & 9 & 3 & 4 & 0.01 & 0.01 & 0.02 & 0.39 & 4 & 4 & 0.08 & 0.47 & $* 0.40$ \\
17 & 9 & 4 & 4 & 0.01 & 0.00 & 0.02 & 1.66 & 4 & 3 & 0.05 & 0.11 & 1.63 \\
18 & 10 & 5 & 0 & 1.42 & 6.71 & 2.20 & $\infty$ & 10 & 4 & 8.32 & 32502.42 & $\infty$ \\
19 & 10 & 9 & 0 & 0.00 & 0.00 & 0.01 & 24.16 & 9 & 5 & 14.54 & 17.32 & 28.75 \\
20 & 11 & 4 & 0 & 0.22 & $* 0.21$ & 7.66 & 2.19 & 29 & $?$ & $\infty$ & $\infty$ & $* 10.61$ \\
\hline
\end{tabular}

symbol $*$ shows the timing which is important in comparison with the other timings. Timings were conducted on a personal computer with $3.2 \mathrm{GHz}$, Intel(R)-Xeon(TM), 1024 MB memory and 64 bits under the Linux operating system.

The experiments we made seem to show that these first implementations are already very efficient. Especially, a comparison of the column time of reg and regIdeal in Table 1 shows that our algorithm to compute the Castelnuovo-Mumford regularity is more efficient than regIdeal (except for Examples 16 and 19). The timings of Examples 1, 7,11 and 12 show that for the ideals which are already in QSP, our algorithm does not run faster than regIdeal. Note that regIdeal algorithm is based on [5] by using the notion of nested type for monomial ideals. It makes a random linear change of variables in $K^{n d-d(d-1) / 2}$ where $d$ is the dimension of input ideal to put the input ideal in this position. Next, it computes the Gröbner basis of the changed ideal (say $I$ ) to test nested type and then computes the satiety of $\widehat{\sec }(\operatorname{in}(I), i)$ for $i=0, \ldots, d$ for computing the regularity.

The efficiency of our algorithm comes from Theorem 4.1] By an incremental (one variable after the other) random linear change of variables it puts a general homogeneous ideal in QSP. Then by computing the Gröbner basis of the changed ideal it tests QSP (which is less expensive than the Borel test and the nested type test). Finally, it reads the regularity without further computation.

As a counterpart of its efficiency, it makes the linear change of variables in $K^{N}$ with $N=n(n+1) / 2-(n-d-1)(n-d) / 2$ which is larger than $K^{n d-d(d-1) / 2}$ (that of [5]). But, in practice, since it makes this change of variables incrementally, the set which is used is less than $K^{N}$ (see the column ncv).

Also, this incremental change of variables makes the computation of Gröbner bases less expensive than the case when we change all the last variables in one step and avoids destroying the possible sparsity and therefore transforming an easy problem into an intractable one (see Example 18 and 20).

This table shows also that sat algorithm is more efficient than satiety $(., 1)$. The last algorithm makes a random linear change of variables to find a monomial ideal of nested type with the same satiety. Once a monomial ideal is nested type, 
one could compute its satiety from its Gröbner basis. The efficiency of our algorithm comes from the fact that we change only the last variable to put the ideal in generic position (see Section 2). But, it is not more efficient than satiety. The SINGUlAR code of satiety is only the following line to compute the satiety of an ideal $I$ :

$$
\text { maxdeg1 (simplify (reduce (quotient (I, maxideal (1)) , I) ,2)) +1; }
$$

The efficiency of this algorithm is followed by using the commands quotient and reduce, which compute, respectively, the quotient ideal of $I$ with respect to the maximal ideal and the normal form of this quotient with respect to $I$.

\section{Conclusion}

In this paper, we have introduced the new notion of a homogeneous ideal in QSP. A definition for the notion of generic coordinates to compute efficiently the Castelnuovo-Mumford regularity of a homogeneous ideal. We have provided an algorithm to decide whether a monomial ideal is in QSP or not which has a quadratic complexity in input (a monomial ideal) size. We have proved that the Castelnuovo-Mumford regularity of an ideal in QSP is the maximal degree of the elements of its reduced Gröbner basis with respect to the reverse lexicographic ordering. Then, we have presented two algorithms sat and reg (using this result) to compute, respectively, the satiety and the Castelnuovo-Mumford regularity of a general homogeneous ideal by an incremental random linear change of variables to put the ideal in a convenient generic position. Our experiments show the efficiency of our algorithms compared with the corresponding algorithms of SINGULAR.

\section{ACKNOWLEDGMENT}

This work was supported in part by the CEAMA, Isfahan University of Technology, Isfahan 84156, Iran. The author would like to thank an anonymous referee for helpful comments.

\section{REFERENCES}

[1] Amir Hashemi. noether.lib. A Singular 3.0.3 distributed library for computing Castelnuovo-Mumford regularity, 2007.

[2] Dave Bayer. The Division Algorithm and the Hilbert Scheme. PhD thesis, Harvard University, 1982.

[3] David Bayer and Michael Stillman. A criterion for detecting m-regularity. Invent. Math., 87(1):1-11, 1987. MR862710 (87k:13019)

[4] Isabel Bermejo and Philippe Gimenez. Computing the Castelnuovo-Mumford regularity of some subschemes of $\mathbb{P}_{K}^{n}$ using quotients of monomial ideals. J. Pure Appl. Algebra, 164(1-2):23-33, 2001. Effective methods in algebraic geometry (Bath, 2000). MR.1854328 (2002i:13013)

[5] Isabel Bermejo and Philippe Gimenez. Saturation and Catelnuovo-Mumford regularity. J. Algebra, 303:592-617, 2006. MR2255124 (2007f:13026)

[6] Wolfram Decker, Gert-Martin Greuel, and Gerhard Pfister. Primary decomposition: algorithms and comparisons. In Algorithmic algebra and number theory (Heidelberg, 1997), pages 187-220. Springer, Berlin, 1999. MR.1672046 (99m:13049)

[7] David Eisenbud. Commutative algebra with a view toward algebraic geometry, volume 150 of Graduate Texts in Mathematics. Springer-Verlag, New York, 1995. MR1322960 (97a:13001)

[8] David Eisenbud and Shiro Goto. Linear free resolutions and minimal multiplicity. J. Algebra, 88(1):89-133, 1984. MR741934 (85f:13023)

[9] Shalom Eliahou and Michel Kervaire. Minimal resolutions of some monomial ideals. J. Algebra, 129(1):1-25, 1990. MR 1037391 (91b:13019) 
[10] André Galligo. À propos du théorème de-préparation de Weierstrass. In Fonctions de plusieurs variables complexes (Sém. François Norguet, octobre 1970-décembre 1973; à la mémoire d'André Martineau), pages 543-579. Lecture Notes in Math., Vol. 409. Springer, Berlin, 1974. MR0402102 (53:5924)

[11] M. Giusti. Some effectivity problems in polynomial ideal theory. In EUROSAM 84 (Cambridge, 1984), volume 174 of Lecture Notes in Comput. Sci., pages 159-171. Springer, Berlin, 1984. MR779123 (86d:12001)

[12] Mark L. Green. Generic initial ideals. In Six lectures on commutative algebra (Bellaterra, 1996), volume 166 of Progr. Math., pages 119-186. Birkhäuser, Basel, 1998. MR.1648665 (99m:13040)

[13] G.-M. Greuel, G. Pfister, and H. Schönemann. Singular 3.0. A Computer Algebra System for Polynomial Computations, Centre for Computer Algebra, University of Kaiserslautern, 2005.

[14] D. Lazard. Gröbner bases, Gaussian elimination and resolution of systems of algebraic equations. In Computer algebra (London, 1983), volume 162 of Lecture Notes in Comput. Sci., pages 146-156. Springer, Berlin, 1983. MR774807 (86m:13002)

[15] Daniel Lazard. Résolution des systèmes d'équations algébriques. Theoret. Comput. Sci., 15(1):77-110, 1981. MR619687 (82i:12001)

Department of Mathematical Sciences, Isfahan University of Technology, Isfahan 84156-83111, IRAN

E-mail address: Amir.Hashemi@cc.iut.ac.ir 